\section{Visual acuity outcomes following surgery for idiopathic epiretinal membrane: an analysis of data from 2001 to 2011}

SR Dawson, M Shunmugam and TH Williamson

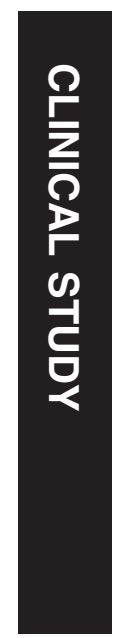

Eye (2014) 28, 219-224; doi:10.1038/eye.2013.253; published online 6 December 2013

Keywords: epiretinal membrane; pars plana vitrectomy; visual acuity

\section{Introduction}

The epiretinal membrane (ERM) is a semitranslucent, glial, fibrocellular membrane found on the inner surface of the internal limiting membrane (ILM) at the macula. They are usually caused by glial proliferation on the surface of the macula and can cause varying degrees of retinal distortion or even cystic macular changes with consequent visual deterioration. They most commonly occur in the elderly population and can cause distortion, blurring of vision, and in some cases severe vision loss. ${ }^{1,2}$ Machemer et $a l^{3}$ were the first to describe pars plana vitrectomy (PPV) with ERM peeling procedures. Several studies have shown this procedure to be beneficial to patients' longterm visual acuity (VA). ${ }^{2}$ Over the course of the last decade, there have been significant improvements in vitreo-retinal techniques leading to reduced complication rates and better outcomes for patients. ${ }^{2}$ Patients with poor preoperative VA have been seen to have more frequent and greater improvement in VA. ${ }^{4}$ However, are better visual outcomes achieved by operating when there is better pre-operative vision? Surgery can lead to complications, which can worsen vision and in some cases cause complete visual loss in the operated eye. ${ }^{5}$ In addition, many ERMs are asymptomatic and non-progressive. Population studies have shown the rate of progression of ERMs to be
Department of Ophthalmology, St. Thomas Hospital, London, UK

Correspondence: Clinic, St. Thomas' Hospital, London SE1 7EH, UK. Tel: $+44(0) 207099$ 0975; Fax: + 44 (0)20 70990974 . E-mail: retinasurgery.co.uk@ gmail.com Accepted in revised form: 12 August 2013 Published online: 6 December 2013 TH Williamson, The Eye

Received: 18 March 2013 
between 16 and $33 \% .{ }^{6}$ ERMs have even been reported to regress in around a quarter of cases. ${ }^{6}$ It is therefore important to identify when to intervene with surgery. Visual acuity is commonly used as an indicator for timing of surgical intervention and its outcomes. In this study, data were analysed to identify the visual outcomes of ERM surgery to try to guide the surgeon on the success rates for this surgery at different levels of presenting VA.

\section{Materials and methods}

Data on consecutive patients presenting with idiopathic ERM to a single surgeon's (THW) vitreoretinal service over a 10-year period were entered prospectively into an electronic patient record (Vitreor available with Vitreoretinal Surgery, Springer). ${ }^{7}$ Data from initial and follow-up clinic visits and intraoperative data were included in the database. The data were then retrospectively reviewed and analysed.

Only the first eye of patients identified with an ERM were included in the study. Pre-operative clinical examination was used to identify the first eye involved. Those with secondary ERM from uveitis, prior retinal detachment, vascular occlusion, diabetic retinopathy, or trauma were excluded. Patients with VMT were not excluded as the authors regard VMT with ERM as a variant of idiopathic ERM. The presence or absence of PVD was noted pre-operatively and the induction of PVD was noted during surgery.

Other recorded data included the laterality of the ERM, duration of symptoms, duration of follow-up, and both pre- and post-operative logMAR VA. In addition, preoperative data on the status of the lens (including the presence of visually significant cataract or pseudophakia) and date of prior cataract surgery were recorded. Perioperative interventions and complications were recorded. Post-operative VA was recorded as a measure of surgical outcome.

All patients underwent standard three-port PPV with or without concurrent small-incision phacoemulsification and intraocular lens implantation. ERM peeling was performed with micro-forceps with the aid of a dye if deemed necessary by the surgeon intra-operatively. Ethical approval for the study was obtained from the local research ethics committee in 2001(Guys \& St Thomas' NHS Foundation Trust). SPSS for Windows version 17 (SPSS Inc., Chicago, IL, USA) was used for data analysis. All data were tested for normality and appropriate statistical tests were used for analysis. Nonparametric tests used included the Chi-squared analysis, the Mann-Whitney $U$-test, and the Jonckheere Terpstra test. The latter was used to assess ordered differences between groups-that is, to assess the presence of an upward or downward trend. A $P$-value $<0.05$ was considered statistically significant.

\section{Results}

Five cases $(1.5 \%)$ were excluded because of insufficient data availability. A total of 237 patients were included in this study in which surgery was performed between 14 February 2001 and 25 October 2011. Of them, 129 (54.4\%) were female with the average age of the cohort being 68.8 years (SD 9.5), ranging from 28.5-94.4 years. The mean spherical equivalent was found to be 0.00 Dioptres (SD 2.51) and the average intraocular pressure of the affected eyes pre-operatively was $15 \mathrm{mmHg}$ (SD 2.60). The median duration of follow-up was 0.55 years (0.32-1.01). The duration of visual loss was available for only 153 patients with a median of 1 year (range $0.12-10$ years). Dye was used in 76 cases: trypan blue was used in 73 cases, brilliant blue was used in 1 case, indocyanine green was used in 1 case, and trypan and brilliant blue were used together in 2 cases. The use of dye was shown to have no effect on the change in VA of patients $(P=0.098$; Mann-Whitney $U)$. Intraoperative variables and complications are illustrated in Table 1.

Pre-operative and post-operative visual acuities are plotted on a scatter graph (linear $R^{2}=0.22, P<0.0001$; Figure 1). Both pre- and post-operative visual acuities were found to be non-normally distributed (ShapiroWilk Test; $P<0.05)$. The median pre-operative logMAR VA was 0.60 (SD 0.48-0.78; Snellen equivalent 6/36) and post-operative was 0.30 (SD $0.18-0.60 ; 6 / 12$ ). The difference was found to be statistically significant (Wilcoxin signed rank; $P<0.005$ ) with the median change in logMAR VA being 0.18 (SD 0.00-0.42).

A total of $165(69.6 \%)$ patients showed an improvement in VA following the procedure; 36 (15.2\%) had no change and $36(15.2 \%)$ had a worse VA postoperatively. The median pre-operative VA for those whose vision worsened was $0.48(6 / 18)$. The demographics for this group were similar to those whose vision remained the same or improved. Eighty patients (34\%) showed an improvement in VA by more than 0.3 LogMAR post-operatively. A total of 168 patients (70.8\%) achieved a post-operative VA of 6/18 or more and 135 patients $(57.0 \%)$ had a post-operative VA of $6 / 12$ or more.

Most patients $(n=201 ; 85 \%)$ were phakic and $36(15 \%)$ were pseudophakic at the time of surgery. An overall 146 patients $(61 \%)$ were observed to have cataract preoperatively. Almost half $(n=125 ; 53 \%)$ had combined PPV and lens extraction; 115 of these patients had cataract and 10 had a normal lens. A third of patients ( $n=76 ; 32.4 \%)$ were phakic at follow-up. The preoperative, post-operative, and change in VA of patients 
who were both phakic and pseudophakic were all found to be non-normally distributed (Shapiro-Wilks; $P<0.05$ ). No significant difference (Mann-Whitney $U$-test; $P=0.27$ ) in change in VA post-operatively was found between patients who were phakic (including those with cataract that was not removed) and patients who were pseudophakic (either had cataract extraction prior to or at the time of operation). No significant difference in change in VA was found between patients who were previously pseudophakic and those who were rendered pseudophakic at the time of ERM peeling (MannWhitney $U$-test; $P=0.84$ ).

Table 1 Intra-operative variables and complications

\begin{tabular}{lcc}
\hline Intra-operative variable & Result & Frequency \\
\hline Anaesthetic & General anaesthesia & $165(69.6 \%)$ \\
& Peribulbar anaesthesia & $40(16.9 \%)$ \\
& Subtenons anaesthesia & $32(13.5 \%)$ \\
Gauge used & 20 & $223(94 \%)$ \\
Dye & 23 & $14(6 \%)$ \\
& None & $160(67.5 \%)$ \\
& Trypan blue & $73(30.8 \%)$ \\
Tamponade & Indocyanine green & $1(0.4 \%)$ \\
& Trypan and Brilliant blue & $2(0.8 \%)$ \\
& Brilliant blue & $1(0.4 \%)$ \\
& 1300 silicone oil & $1(0.4 \%)$ \\
& AIR & $28(11.8 \%)$ \\
& C3F8 & $5(2.1 \%)$ \\
Complications & Nil & $177(74.7 \%)$ \\
& SF6 & $26(11.0 \%)$ \\
& Iatrogenic tears & $21(8.9 \%)$ \\
& Choroidal haemorrhage & 0 \\
& Lens touch & $5(2.1 \%)$ \\
\hline
\end{tabular}

To further explore the importance of pre-operative VA on outcome, this variable was used to categorise patients into three groups: VA $0.5(6 / 19)$ or better, VA 0.6-0.9 (6/24-6/48), and VA $1.0(6 / 60)$ or worse. The number of patients in each of the groups and a summary of medians and interquartile ranges can be seen in Table 2. Overall, post-operative VA was found to be greatest in those who had a pre-operative VA of 0.5 or better (median VA 0.18 , range $0.14-0.30$ ), followed by those with a pre-operative VA of 0.6-0.9 (median VA 0.48, range 0.18-0.60). Those with a pre-operative VA of 1.0 or worse had the lowest median post-operative VA (median 0.69, range 0.30-1.0). However, when measuring the change in VA the number of patients with an improvement in logMar VA of $>0.3$ was greatest in those who had a pre-operative VA of 1.0 or worse (median change in VA 0.61 , range $0.0-0.83$ ), followed by those in the range of 0.6-0.9 (median change in VA 0.30 , range $0.0-0.42$ ). The group of patients whose pre-operative VA was 0.5 or better had the least proportion of patients to improve by $>0.3$ (median change VA 0.12 , range 0.0-0.30) (Chi-squared analysis; $P<0.001)$.

The data were then categorised according to the year in which surgery had taken place. Median pre-operative VA, post-operative VA, and change in VA between 2001 and 2011 can be seen in Table 3. A statistically significant increase was found between the median change in visual acuities in order from 2001 to 2011; that is, patients operated upon in the more recent years had statistically greater change in VA than those operated upon in earlier years (Jonckheere Terpstra Test; $P=0.004$ ). The median post-operative VA also improved from 2001 to 2011 (Jonckheere Terpstra; $P=0.001$ ). There was no change in pre-operative VA (Jonckheere Terpstra; $P=0.022$ ).

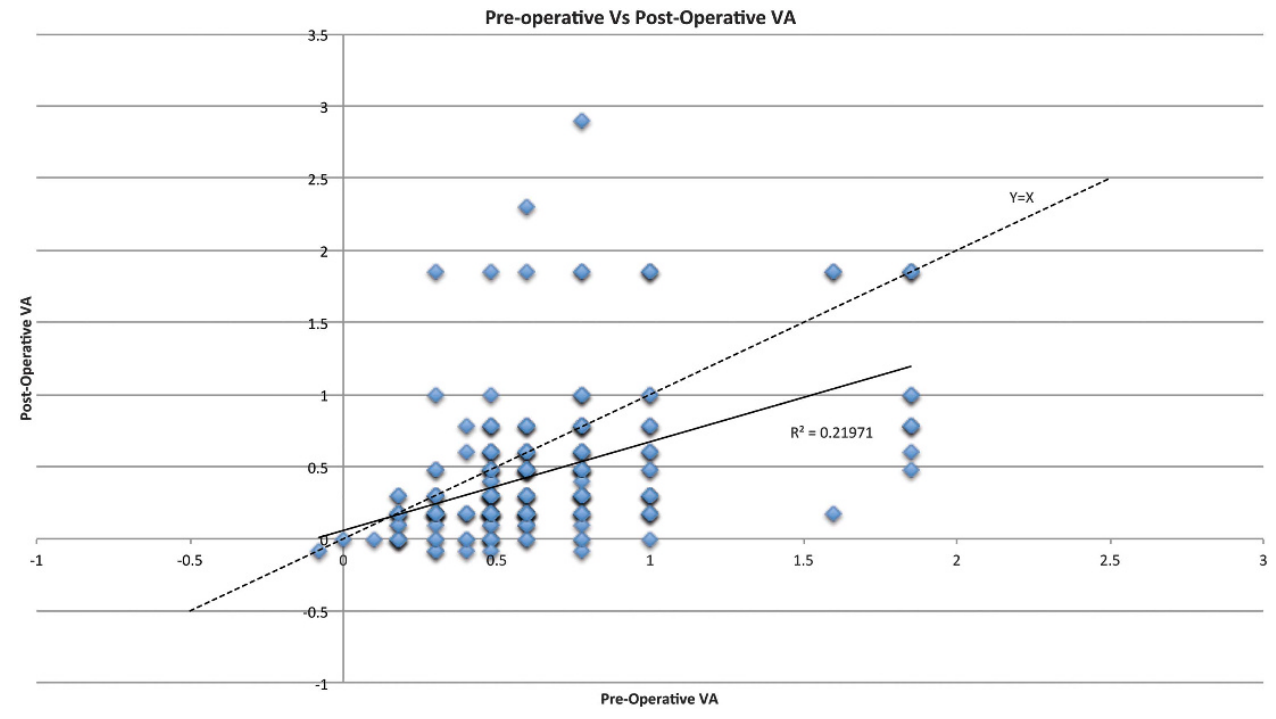

Figure 1 Scatter plot of pre-operative versus post-operative visual acuity. VA, visual acuity. 
Table 2 Median pre-operative, post-operative, and VA changes of group

\begin{tabular}{lcccc}
\hline Pre-operative VA ranges & Number of cases, $\mathrm{n}(\%)$ & $\begin{array}{c}\text { Median pre-operative VA, } \\
\text { LogMAR }\end{array}$ & $\begin{array}{c}\text { Median post-operative VA, } \\
\text { LogMAR }\end{array}$ & $\begin{array}{c}\text { Patients with VA improvement } \\
>0.3, \mathrm{n}(\%)\end{array}$ \\
\hline VA 0.5 or better & $111(46.8)$ & $0.48(0.30-0.48)$ & $0.18(0.14-0.3)$ & 15 out of $111(13.5 \%)$ \\
VA 0.6-0.9 & $90(40.0)$ & $0.60(0.60-0.78)$ & $0.48(0.18-0.60)$ & 43 out of $90(47.8 \%)$ \\
VA 1.0 or worse & $36(15.2)$ & $1.0(1.0-1.85)$ & $0.69(0.30-1.0)$ & 23 out of $36(63.8 \%)$ \\
\hline
\end{tabular}

Abbreviation: VA, visual acuity.

Table 3 Median pre-operative, post-operative, and change in VA categorised by study year

\begin{tabular}{cccccc}
\hline Year & Number of patients (\%) & $\begin{array}{c}\text { Median Pre-op VA, } \\
\text { LogMAR }\end{array}$ & $\begin{array}{c}\text { Median post operative VA, } \\
\text { LogMAR }\end{array}$ & $\begin{array}{c}\text { Change in VA median, } \\
\text { LogMAR }\end{array}$ & $\begin{array}{c}\text { Patients with } \Delta \text { VA }>0.3, \\
\mathrm{n}(\%)\end{array}$ \\
\hline 2001 & $18(7.6)$ & $0.60(0.47-0.77)$ & $0.39(0.18-0.57)$ & $0.15(0.00-0.39)$ & $5(27.8)$ \\
2002 & $24(10.1)$ & $0.69(0.48-0.78)$ & $0.48(0.27-0.78)$ & $0.12(0.03-0.30)$ & $4(16.7)$ \\
2003 & $10(4.2)$ & $0.54(0.47-0.77)$ & $0.69(0.51-0.77)$ & $-0.06(-0.21-0.14)$ & $1(10.0)$ \\
2004 & $21(8.9)$ & $0.77(0.47-1.00)$ & $0.47(0.18-0.78)$ & $0.18(0.00-0.42)$ & $7(33.3)$ \\
2005 & $25(10.5)$ & $0.47(0.47-0.77)$ & $0.47(0.18-0.60)$ & $0.30(0.00-0.40)$ & $10(40.0)$ \\
2006 & $18(7.6)$ & $0.60(0.47-0.77)$ & $0.18(0.18-0.53)$ & $0.18(0.12-0.48)$ & $8(44.4)$ \\
2007 & $24(10.1)$ & $0.47(0.30-0.60)$ & $0.18(0.16-0.33)$ & $0.18(0.11-0.41)$ & $9(37.5)$ \\
2008 & $27(11.4)$ & $0.47(0.47-0.77)$ & $0.30(0.18-0.48)$ & $0.30(0.00-0.36)$ & $9(33.3)$ \\
2009 & $29(12.2)$ & $0.60(0.30-0.60)$ & $0.18(0.18-0.48)$ & $0.12(0.00-0.42)$ & $10(34.5)$ \\
2010 & $27(11.4)$ & $0.47(0.40-0.60)$ & $0.30(0.18-0.39)$ & $0.30(0.12-0.45)$ & $11(40.7)$ \\
2011 & $14(5.9)$ & $0.60(0.47-1.00)$ & $0.24(0.18-0.53)$ & $0.41(0.19-0.65)$ & $8(57.1)$ \\
\hline
\end{tabular}

Abbreviation: VA, visual acuity.

Table 4 List and corresponding incidence of post-operative complications in study cohort

\begin{tabular}{lcc}
\hline Complication & Number of patients in cohort analysis, $\mathrm{n}$ & Incidence of complication, $\mathrm{n}(\%)$ \\
\hline Recurrence of ERM & 237 & $3(1.3 \%)$ \\
Glaucoma & 237 & $5(2.1 \%)$ \\
Cataract & 75 & $21(28 \%) 7$ of these were present before surgery \\
Retinal detachment & 237 & $2(0.8 \%)$ \\
Phthisis & 237 & $1(0.4 \%)$ \\
\hline
\end{tabular}

Post-operative complications are shown in Table 4 and include retinal detachment in two eyes $(0.8 \%)$, one phthisis $(0.4 \%)$, and three recurrences of ERMs $(1.3 \%)$ at follow-up.

\section{Discussion}

ERMs are commonly idiopathic, following a posterior vitreous detachment, or can be secondary to a number of retinal disorders including uveitis, trauma, prior surgery, or retinal breaks. Idiopathic ERM incidence increases with age from less than $4 \%$ in those under 60 years to $20 \%$ in those over 75 years, as reported in one autopsy study. 6,8

The results of this study demonstrate improvement in visual acuity after surgery for ERM. The largest improvement in vision was found in those patients with the worst pre-operative VA. This contrasts with other conditions of the macula in which interventions become ineffective once the VA deteriorates severely-for example, diabetic maculopathy and age-related macular degeneration. ${ }^{9-11}$ It should be noted that those with better initial VA have to gain less to achieve optimal VA compared with those with worse initial VA; therefore, this ceiling effect may be a factor in the comparably greater change in VA in the latter group.

The regression line in Figure 1 diverges from the unit line more markedly for patients with poorer preoperative VA. This corresponds to the clinical assumption of patients experiencing a greater improvement in VA when operated on at a level of poorer pre-operative VA. The intersection between the unit and regression lines is very close to zero and so it would, on average, appear that all except those patients with LogMAR VA better than 0.1 would improve with surgery. It should also be noted, however, that, even though VA may remain unchanged for many patients close to the unit line, perceived visual quality may improve as ERM removal also improves symptoms of metamorphopsia. 
The data demonstrate that presenting VA did not change over time; however, final VA outcomes have improved from 2001 to 2011. The reasons for this are not clear, but a number of changes in management occurred during this period-for example, Optical Coherence Tomography (OCT), which had been used in St Thomas' since 1995, changing to Fourier-domain OCT in 2010. Adjunctive OCT scanning objectively provides the examiner with evidence of ERMs, which may not have been clinically detectable on biomicroscopy alone. OCT displays ERMs as a thin, smooth, hyper-reflective line along the surface of the retina and allows the examination of secondary changes in the retina, such as cystoid macular oedema and loss of the inner segment/ outer segment line and external limiting membrane. Improved OCT imaging may have led to better identification of ERMs and their effects on the retina, influencing outcomes of surgery, and may account for some of the improvement in outcomes in the later years but the numbers in the subcategories are too small to confirm this. Currently, using discriminant functions based on pre-operative VA and duration of visual blur alone yields up to $80 \%$ accuracy when classifying eyes into a group showing improvement in VA. ${ }^{12,13}$

The use of dye is unlikely to be a factor in the improvement in outcomes as no difference was found in change in VA when a comparison was made between the presence or absence of dye. Both surgical instrumentation and technique remained stable throughout the study and the criteria for operating on ERMs have not changed. Our numbers of small-gauge PPV surgeries were regarded as too small to allow assessment of that variable. However, other studies have shown quicker visual recovery and fewer complications with small-gauge trans-conjunctival sutureless vitrectomy techniques for ERM removal. ${ }^{14-16}$

One of the limitations of this study was the use of VA as the sole measure of surgical success. Metamorphopsia is also a significant contributor to morbidity in patients with ERMs and it has been shown that, even though VA alone may not improve following ERM removal, metamorphopsia and quality of life does. ${ }^{13}$ The results from this study show that ERM peeling is an effective treatment modality in improving VA in patients with ERMs with $70 \%$ achieving an improvement and more than half having a post-operative VA of 6 out of 12 or better. On the basis of previous studies, it is known that the functional improvement is likely to be higher than the documented VA improvement. ${ }^{13}$ Furthermore, ERMs are known to cause aniseikonia with improvement in stereoacuity following ERM removal, especially if performed early. ${ }^{17,18}$

There is conflicting opinion regarding the timing of ERM removal. This study, one of the largest published series, demonstrates that earlier intervention (better preoperative VA) yields a better final VA. This relationship was linear with $R^{2}=0.22(P<0.0001$; Figure 1$)$. The high level of variance shown by this $R^{2}$ value is likely due to other conditions within the retina, such as loss of receptors, cystoid macular oedema, or surgical trauma, all of which further reduce vision.

Other limitations of the study include its retrospective design and the variable time between operation date and follow-up for individual patients. To improve the reliability of the results in future studies, patients could be assessed and data recorded at fixed time points-for example, at 2 and 6 months.

The inclusion of patients both with and without cataract pre-operatively and post-operatively is also a limitation. However, by including these cases, the real population of patients with ERM has been represented. Combined cataract extraction and PPV can confound analysis of VA in vitreoretinal surgery. However, our analysis of the effects of pseudophakia and cataract removal did not show an effect on comparative VA outcomes in this study. The rate of complications was also similar between those undergoing ERM peeling only and those undergoing the combined procedure. Rates of complications in this study were similar to those of previously published articles. ${ }^{5}$ It would therefore be of economic and symptomatic benefit to the patient if a combined procedure was undertaken as the rates of cataract progression are high following PPV. ${ }^{19,20}$

In conclusion, this study shows improvement in VA after PPV and ERM removal. Patients with better initial VA achieve higher levels of visual outcome but those with poorer pre-operative VA show a greater change in VA following ERM surgery. Results of surgery also improved over the time period of the study.

\section{Conflict of interest}

The authors declare no conflict of interest.

\section{Author contributions}

All authors have made substantial contributions to conception and design, acquisition of data, and analysis and interpretation of data. They have also been involved in drafting the article and have approved the final version to be published.

\section{References}

1 Jacobsen CH. Epiretinal membranes. Optom Clin 1996; 5(1): 77-94.

2 Wong JG, Sachdev N, Beaumont PE, Chang AA. Visual outcomes following vitrectomy and peeling of epiretinal membrane. Clin Experiment Ophthalmol 2005; 33(4): 373-378. 
3 Machemer R. [The surgical removal of epiretinal macular membranes (macular puckers) (author's transl)]. Klin Monbl Augenheilkd 1978; 173(1): 36-42.

4 Pournaras CJ, Emarah A, Petropoulos IK. Idiopathic macular epiretinal membrane surgery and ILM peeling: anatomical and functional outcomes. Semin Ophthalmol 2011; 26(2): 42-46.

5 Guillaubey A, Malvitte L, Lafontaine PO, Hubert I, Bron A, Berrod JP et al. Incidence of retinal detachment after macular surgery: a retrospective study of 634 cases. Br J Ophthalmol 2007; 91(10): 1327-1330.

6 Fraser-Bell S, Guzowski M, Rochtchina E, Wang JJ, Mitchell P. Five-year cumulative incidence and progression of epiretinal membranes: the Blue Mountains Eye Study. Ophthalmology 2003; 110(1): 34-40.

7 Williamson TH. Vitreoretinal Surgery. Springer: Berlin; London, 2007.

8 Roth AM, Foos RY. Surface wrinkling retinopathy in eyes enucleated at autopsy. Trans Am Acad Ophthalmol Otolaryngol 1971; 75(5): 1047-1058.

9 Flaxel CJ, Edwards AR, Aiello LP, Arrigg PG, Beck RW, Bressler NM. Factors associated with visual acuity outcomes after vitrectomy for diabetic macular edema: diabetic retinopathy clinical research network. Retina 2010; 30(9): 1488-1495.

10 Schulze SD, Hesse L. Tissue plasminogen activator plus gas injection in patients with subretinal hemorrhage caused by age-related macular degeneration: predictive variables for visual outcome. Graefes Arch Clin Exp Ophthalmol 2002; 240(9): 717-720.

11 Smiddy WE. Clinical applications of cost analysis of diabetic macular edema treatments. Ophthalmology 2012; 119(12): 2558-2562.
12 Rice TA, De Bustros S, Michels RG, Thompson JT, Debanne SM, Rowland DY. Prognostic factors in vitrectomy for epiretinal membranes of the macula. Ophthalmology 1986; 93(5): 602-610.

13 Ghazi-Nouri SM, Tranos PG, Rubin GS, Adams ZC, Charteris DG. Visual function and quality of life following vitrectomy and epiretinal membrane peel surgery. Br J Ophthalmol 2006; 90(5): 559-562.

14 Valmaggia C. Pars plana vitrectomy with 25-gauge instruments in the treatment of idiopathic epiretinal membranes. Klin Monbl Augenheilkd 2007; 224(4): 292-296.

15 Rizzo S, Genovesi-Ebert F, Murri S, Belting C, Vento A, Cresti $\mathrm{F}$ et al. 25-gauge, sutureless vitrectomy and standard 20-gauge pars plana vitrectomy in idiopathic epiretinal membrane surgery: a comparative pilot study. Graefes Arch Clin Exp Ophthalmol 2006; 244(4): 472-479.

16 Konstantinidis L, Berguiga M, Beknazar E, Wolfensberger TJ. Anatomic and functional outcome after 23-gauge vitrectomy, peeling, and intravitreal triamcinolone for idiopathic macular epiretinal membrane. Retina 2009; 29(8): 1119-1127.

17 Asaria R, Garnham L, Gregor ZJ, Sloper JJ. A prospective study of binocular visual function before and after successful surgery to remove a unilateral epiretinal membrane. Ophthalmology 2008; 115(11): 1930-1937.

18 Ugarte M, Williamson TH. Aniseikonia associated with epiretinal membranes. Br J Ophthalmol 2005; 89(12): 1576-1580.

19 Alexandrakis G, Chaudhry NA, Flynn Jr HW, Murray TG. Combined cataract surgery, intraocular lens insertion, and vitrectomy in eyes with idiopathic epiretinal membrane. Ophthalmic Surg Lasers 1999; 30(4): 327-328.

20 Cherfan GM, Michels RG, de Bustros S, Enger C, Glaser BM. Nuclear sclerotic cataract after vitrectomy for idiopathic epiretinal membranes causing macular pucker. Am J Ophthalmol 1991; 111(4): 434-438. 